\title{
Possible Reentrance of the Fractional Quantum Hall Effect in the Lowest Landau Level
}

\author{
M. O. Goerbig ${ }^{1,2}$, P. Lederer ${ }^{2}$, and C. Morais Smith ${ }^{1,3}$ \\ ${ }^{1}$ Département de Physique, Université de Fribourg, Pérolles, CH-1700 Fribourg, Switzerland. \\ ${ }^{2}$ Laboratoire de Physique des Solides, Bat.510, UPS (associé au CNRS), F-91405 Orsay cedex, France. \\ ${ }^{3}$ Institute for Theoretical Physics, Utrecht University, \\ Leuvenlaan 4, 3584 CE Utrecht, The Netherlands.
}

\begin{abstract}
In the framework of a recently developed model of interacting composite fermions, we calculate the energy of different solid and Laughlin-type liquid phases of spin-polarized composite fermions. The liquid phases have a lower energy than the competing solids around the electronic filling factors $\nu=4 / 11,6 / 17$, and 4/19 and may thus be responsible for the fractional quantum Hall effect at $\nu=4 / 11$. The alternation between solid and liquid phases when varying the magnetic field may lead to reentrance phenomena in analogy with the observed reentrant integral quantum Hall effect.

PACS numbers: 73.43.Nq, 71.10.Pm, 73.20.Qt
\end{abstract}

Recent experiments by Eisenstein et al. have revealed a reentrance of the integer quantum Hall effect (IQHE) when the two spin branches of the lowest Landau level (LL) are completely filled and the first excited LL $(n=1)$ starts to be populated [1]. This phenomenon arises when the partial filling of the topmost LL is varied, and it is due to an alternation of electron-solid phases, such as the Wigner crystal (WC) or a bubble crystal, and quantum liquids, which display the fractional quantum Hall effect (FQHE) 2]. Both liquid and solid phases have their origin in the Coulomb interaction between the electrons, which is the relevant energy scale when the last occupied LL is not completely filled. In this case, intra-LL excitations coupling states with the same kinetic energy are possible, in contrast to the case of completely filled LLs, where such excitations are prohibited by the Pauli principle. Electrons in the completely filled lower LLs may then be considered as an inert homogeneous background.

The FQHE in the lowest LL occurs at the filling factors $\nu=n_{e l} / n_{B}=p /(2 p s+1)$, where $n_{e l}$ denotes the electronic and $n_{B}=e B / h$ the flux density. It may be interpreted as an IQHE of a new type of particle, a so-called composite fermion (CF) [3], which consists of an electron and a vortex-like object, which carries $s$ pairs of flux quanta. Because of its reduced coupling $(e B)^{*}=e B /(2 p s+1)$ to the magnetic field $B, p$ CFLLs are completely filled when $\nu^{*}=p$, where the CFLL filling $\nu^{*}$ factor is related to the electronic one by $\nu=\nu^{*} /\left(2 s \nu^{*}+1\right)$. The similarity between the IQHE and the FQHE naturally raises the question whether a phenomenon analogous to the reentrant IQHE may occur for CFs when a higher CF-LL $(p \geq 1)$ is partially filled. In this case, residual interactions between CFs may lead to the formation of CF-solid phases, such as stripes and bubbles [4], or to incompressible liquids, which may be interpreted in terms of a second generation of $\mathrm{CFs}$ $\left(\mathrm{C}^{2} \mathrm{Fs}\right)$ [5, 6, 7]. The existence of an incompressible $\mathrm{CF}$ liquid is supported by the recent observation of a FQHE at $\nu=4 / 11$, which corresponds to a CF filling factor $\nu^{*}=1+1 / 3$, by Pan et al. 7]. Such a state had been con- jectured before by Mani and v. Klitzing based on arguments concerning the self-similarity of the Hall-resistance curve [8].

In this Letter, we investigate several spin-polarized CF-solid and liquid states in a recently developed model of interacting CFs in a partially filled CF-LL [5], which incorporates the self-similarity of the FQHE [8] by an iterative projection of the dynamics to a single CF-LL. The form of the CF interaction has been derived in the framework of the Hamiltonian theory, proposed by Murthy and Shankar 9]. The basic assumptions are that the degenerate CF-LLs remain stable in the presence of the residual $\mathrm{CF}$ interactions and that the low-energy degrees of freedom are described by intra-level excitations, in agreement with complementary studies in the wavefunction approach [4, 10, 11]. The energies of the competing phases are calculated directly in the thermodynamic limit. In contrast to prior investigations by Lee et al. [4], our energy calculations suggest the possibility of incompressible Laughlin-type liquid states of CFs. Such states have a lower energy than CF solids at certain filling factors also in higher CF-LLs $(p \geq 1)$, e.g. at $\nu=4 / 11,6 / 17,4 / 19$, and $11 / 27$. An alternation between these liquid and $\mathrm{CF}$-solid phases with varying $\mathrm{CF}$ filling occurs in our energy studies and may lead to observable reentrance phenomena in the FQHE regime.

The model Hamiltonian of CFs, the dynamics of which are restricted to the $p$-th CF-LL, is given by [5]

$$
\hat{H}(s, p)=\frac{1}{2 A} \sum_{\mathbf{q}} v_{s, p}^{C F}(q) \bar{\rho}^{C F}(-\mathbf{q}) \bar{\rho}^{C F}(\mathbf{q}),
$$

with the measure $\sum_{\mathbf{q}}=A \int d^{2} q /(2 \pi)^{2}$ in terms of the total area $A$ and the CF-interaction potential $\left(l_{B} \equiv 1\right)$

$$
\begin{aligned}
v_{s, p}^{C F}(q)= & \frac{2 \pi e^{2}}{\epsilon q} e^{-q^{2} l_{B}^{* 2} / 2}\left[L_{p}\left(\frac{q^{2} l_{B}^{* 2} c^{2}}{2}\right)\right. \\
& \left.-c^{2} e^{-q^{2} / 2 c^{2}} L_{p}\left(\frac{q^{2} l_{B}^{* 2}}{2 c^{2}}\right)\right]^{2},
\end{aligned}
$$

where $c^{2}=2 p s /(2 p s+1)$ is the vortex charge, $l_{B}^{*}=$ 
$1 / \sqrt{1-c^{2}}$ is the $\mathrm{CF}$ magnetic length, and $L_{p}(x)$ denotes a Laguerre polynomial [9]. The potential takes into account the form of CFs in the $p$-th CF-LL. The projected CF-density operators satisfy the GirvinMacDonald-Platzman algebra 12], $\left[\bar{\rho}^{C F}(\mathbf{q}), \bar{\rho}^{C F}(\mathbf{k})\right]=$ $2 i \sin \left[(\mathbf{q} \times \mathbf{k})_{z} l_{B}^{* 2} / 2\right] \bar{\rho}^{C F}(\mathbf{q}+\mathbf{k})$, if one replaces the electronic by the $\mathrm{CF}$ magnetic length. This model of interacting CFs is similar to the one of interacting electrons in a single LL, and this property allows for the use of the standard theoretical approaches to describe the different phases. Note that we consider an ideal two-dimensional electron gas. Finite-width and impurity effects are not taken into account. In contrast to Ref. [5], where the activation gaps of $\mathrm{C}^{2} \mathrm{~F}$ states have been discussed, we neglect inter-CF-LL mixing, which may in principle be included in a dielectric function and which screens the $\mathrm{CF}$ interaction potential [5].

The energies of the different CF-solid phases are calculated in the Hartree-Fock approximation (HFA), in analogy with the electron-solid phases in higher LLs [2, 13, 14]. We concentrate on triangular bubble crystals with $M$ CFs per bubble and CF-stripe phases, which become relevant at half-filled levels. The CF-WC is formally treated as a bubble crystal with $M=1$. The Hamiltonian (11) may be rewritten in the HFA,

$$
\hat{H}_{H F}(s, p)=\frac{1}{2 A} \sum_{\mathbf{q}} u_{s, p}^{H F}(q)\left\langle\bar{\rho}^{C F}(-\mathbf{q})\right\rangle \bar{\rho}^{C F}(\mathbf{q}),
$$

with the Hartree-Fock potential

$$
u_{s, p}^{H F}(q)=v_{s, p}^{C F}(q)-\frac{1}{n_{B}^{*} A} \sum_{\mathbf{p}} v_{s, p}^{C F}(p) e^{-i\left(p_{x} q_{y}-p_{y} q_{x}\right) l_{B}^{* 2}},
$$

where $n_{B}^{*}=1 / 2 \pi l_{B}^{* 2}$ is the renormalized flux density. The second term takes into account quantum-mechanical exchange effects. The average $\left\langle\bar{\rho}^{C F}(\mathbf{q})\right\rangle$ plays the role of an order parameter, $\Delta^{*}(\mathbf{q})=\left\langle\bar{\rho}^{C F}(\mathbf{q})\right\rangle / n_{B}^{*} A=$ $\int d^{2} r \bar{\nu}^{*}(\mathbf{r}) e^{i \mathbf{q} \cdot \mathbf{r}} / A$, and is related to the local CF filling factor $\bar{\nu}^{*}(\mathbf{r})$ of the $p$-th CF-LL by Fourier transformation. In terms of the order parameter, the cohesive energy $E_{c o h}=\left\langle\hat{H}_{H F}(s, p)\right\rangle / \bar{\nu}^{*} n_{B}^{*} A$ of the CF-solid phases, with the partial CF filling factor $\bar{\nu}^{*}=A^{-1} \int d^{2} r \bar{\nu}^{*}(\mathbf{r})$, is

$$
E_{c o h}^{C F-\operatorname{solid}}\left(s, p ; \bar{\nu}^{*}\right)=\frac{n_{B}^{*}}{2 \bar{\nu}^{*}} \sum_{\mathbf{q}} u_{s, p}^{H F}(q)\left|\Delta^{*}(\mathbf{q})\right|^{2} .
$$

The order parameter for the CF-bubble crystal is given in terms of the Bessel function $J_{1}(x)$,

$$
\Delta_{B}^{*}(\mathbf{q})=\frac{2 \pi \sqrt{2 M} l_{B}^{*}}{A q} J_{1}\left(q \sqrt{2 M} l_{B}^{*}\right) \sum_{j} e^{i \mathbf{q} \cdot \mathbf{R}_{j}},
$$

where $\mathbf{R}_{j}$ are the lattice vectors of the triangular lattice with a lattice spacing $\Lambda_{B}=\left(4 \pi M / \sqrt{3} \bar{\nu}^{*}\right)^{1 / 2} l_{B}^{*}$. One thus obtains for the cohesive energy of the CF-bubble crystal

$$
E_{c o h}^{C F-B}\left(s, p, M ; \bar{\nu}^{*}\right)=\frac{n_{B}^{*} \bar{\nu}^{*}}{M} \sum_{\mathbf{G}_{l} \neq 0} u_{s, p}^{H F}\left(\mathbf{G}_{l}\right) \frac{J_{1}^{2}\left(\sqrt{2 M}\left|\mathbf{G}_{l}\right| l_{B}^{*}\right)}{\left|\mathbf{G}_{l}\right|^{2} l_{B}^{* 2}}
$$

where $\mathbf{G}_{l}$ are the vectors of the reciprocal lattice. The order parameter of the CF-stripe phase,

$$
\Delta_{S}^{*}(\mathbf{q})=\frac{2}{L_{x}} \delta_{q_{y}, 0} \frac{\sin \left(q_{x} \Lambda_{S} \bar{\nu}^{*} / 2\right)}{q_{x}} \sum_{j} e^{i q_{x} j \Lambda_{S}},
$$

with the system extension $L_{x}$ in the $x$-direction, yields the cohesive energy

$E_{c o h}^{C F-S}\left(s, p, \Lambda_{S} ; \bar{\nu}^{*}\right)=\frac{n_{B}^{*}}{2 \pi^{2} \bar{\nu}^{*}} \sum_{j \neq 0} u_{s, p}^{H F}\left(q=\frac{2 \pi}{\Lambda_{S}} j\right) \frac{\sin ^{2}\left(\pi \bar{\nu}^{*} j\right)}{j^{2}}$.

The stripe periodicity $\Lambda_{S}$ is a variational parameter with respect to which the energy is minimized. It scales with the CF cyclotron radius $R_{C}^{*}=l_{B}^{*} \sqrt{2 p+1}$, and one finds $\Lambda_{S}(s=1, p=1)=1.95 R_{C}^{*}, \Lambda_{S}(s=1, p=2)=1.8 R_{C}^{*}$, and $\Lambda_{S}(s=2, p=1)=1.9 R_{C}^{*}$ for the optimal stripe periodicity at $\bar{\nu}^{*}=1 / 2$.

The cohesive energy of the CF solids has to be compared to the energy of Laughlin-type quantum liquids, which may occur around the "magical" filling factors $\bar{\nu}^{*}=1 /(2 \tilde{s}+1)$, with integral $\tilde{s}$. Because of their strong correlations, these liquid phases cannot be treated in the HFA, and one has to use Laughlin's wave functions [15], generalized to an arbitrary LL [16]. Their cohesive energy is given by

$$
E_{c o h}^{L}(s, p ; \tilde{s})=\frac{\bar{\nu}^{*}}{\pi} \sum_{m=0}^{\infty} c_{2 m+1}^{\tilde{s}} V_{2 m+1}(s, p),
$$

with Haldane's pseudopotentials 17] $V_{2 m+1}(s, p)=$ $(2 \pi / A) \sum_{\mathbf{q}} v_{s, p}^{C F}(q) L_{2 m+1}\left(q^{2} l_{B}^{* 2}\right) \exp \left(-q^{2} l_{B}^{* 2} / 2\right)$, and the expansion coefficients $c_{2 m+1}^{\tilde{s}}$ characterize the wave function. In contrast to Ref. [4], where a few number of pseudopotentials, which have been determined numerically, were used to construct a CF interaction potential, here, they are obtained to arbitrary order from the analytical expression of the CF interaction potential (2). As pointed out by the authors of Ref. [4], the construction of an interaction potential from pseudopotentials is not unique. The expansion coefficients $c_{2 m+1}^{\tilde{s}}$ are derived from sum rules [12, 18], which are considered as a set of linear equations [19]. This method yields results deviating less than $1 \%$ from numerical studies and is thus sufficiently accurate for the present investigations.

Away from the magical filling factors, the energy of the CF-liquid phases is raised by the excited quasi-particles [for $\left.\bar{\nu}^{*}>1 /(2 \tilde{s}+1)\right]$ or quasi-holes [for $\left.\bar{\nu}^{*}<1 /(2 \tilde{s}+1)\right]$, which are separated by a gap from the incompressible liquid state. They may be interpreted as $\mathrm{C}^{2} \mathrm{Fs}$ or $\mathrm{C}^{2} \mathrm{~F}$ holes promoted to the next higher $\mathrm{C}^{2} \mathrm{~F}$ level [5, [6] . Their energy is calculated analytically in the framework of the Hamiltonian theory [9]. One finds

$$
\Delta_{s, p}^{q p}(\tilde{s}, \tilde{p})=\frac{1}{2 A} \sum_{\mathbf{q}} v_{s, p}^{C F}(q)\langle\tilde{p}|\overline{\bar{\rho}}(-\mathbf{q}) \overline{\bar{\rho}}(\mathbf{q})| \tilde{p}\rangle
$$




$$
-\frac{1}{A} \sum_{\mathbf{q}} v_{s, p}^{C F}(q) \sum_{j^{\prime}=0}^{\tilde{p}-1}\left|\left\langle\tilde{p}|\overline{\bar{\rho}}(\mathbf{q})| j^{\prime}\right\rangle\right|^{2}
$$

for the quasi-particle energies and

$$
\begin{aligned}
\Delta_{s, p}^{q h}(\tilde{s}, \tilde{p})= & -\frac{1}{2 A} \sum_{\mathbf{q}} v_{s, p}^{C F}(q)\langle\tilde{p}-1|\overline{\bar{\rho}}(-\mathbf{q}) \overline{\bar{\rho}}(\mathbf{q})| \tilde{p}-1\rangle \\
& +\frac{1}{A} \sum_{\mathbf{q}} v_{s, p}^{C F}(q) \sum_{j^{\prime}=0}^{\tilde{p}-1}\left|\left\langle\tilde{p}-1|\overline{\bar{\rho}}(\mathbf{q})| j^{\prime}\right\rangle\right|^{2}
\end{aligned}
$$

for the quasi-hole energies, where the matrix elements $\left(j \geq j^{\prime}\right)$ are given by

$$
\begin{array}{r}
\left\langle j|\overline{\bar{\rho}}(\mathbf{q})| j^{\prime}\right\rangle=\sqrt{\frac{j^{\prime} !}{j !}}\left(\frac{-i\left(q_{x}-i q_{y}\right) \tilde{l} \tilde{c}}{\sqrt{2}}\right)^{j-j^{\prime}} e^{-q^{2} \tilde{l}^{2} \tilde{c}^{2} / 4} \\
\times\left[L_{j^{\prime}}^{j-j^{\prime}}\left(\frac{q^{2} \tilde{l}^{2} \tilde{c}^{2}}{2}\right)-\tilde{c}^{2\left(1-j+j^{\prime}\right)} e^{-q^{2} / 2 \tilde{c}^{2}} L_{j^{\prime}}^{j-j^{\prime}}\left(\frac{q^{2} \tilde{l}^{2}}{2 \tilde{c}^{2}}\right)\right],
\end{array}
$$

with the $\mathrm{C}^{2} \mathrm{~F}$ magnetic length $\tilde{l}=l_{B}^{*} / \sqrt{1-\tilde{c}^{2}}$, in terms of the $\mathrm{C}^{2} \mathrm{~F}$-vortex charge $\tilde{c}^{2}=2 \tilde{p} \tilde{s} /(2 \tilde{p} \tilde{s}+1)[5]$. Here, we are interested only in states from the Laughlin series $(\tilde{p}=1)$, and one finds

\begin{tabular}{|l||c|c||c|c|}
\hline & $\Delta^{q p}(\tilde{s}=1)$ & $\Delta^{q p}(\tilde{s}=2)$ & $\Delta^{q h}(\tilde{s}=1)$ & $\Delta^{q h}(\tilde{s}=2)$ \\
\hline \hline$s=1, p=1$ & 0.04172 & 0.03065 & -0.01567 & -0.01309 \\
\hline$s=1, p=2$ & 0.02330 & 0.01958 & -0.01277 & -0.00967 \\
\hline$s=2, p=1$ & 0.01727 & 0.01391 & -0.00859 & -0.00672 \\
\hline
\end{tabular}

in units of $e^{2} / \epsilon l_{B}$. The cohesive energy of the CF-liquid phases thus becomes

$$
\begin{aligned}
E_{c o h}^{C F-l i q}\left(s, p ; \tilde{s}, \bar{\nu}^{*}\right)= & E_{c o h}^{L}(s, p ; \tilde{s}) \\
& +\left[\bar{\nu}^{*}(2 \tilde{s}+1)-1\right] \Delta_{s, p}^{q p / q h}(\tilde{s}, \tilde{p}=1)
\end{aligned}
$$

where the residual $\mathrm{C}^{2} \mathrm{~F}$ interactions have been neglected. This approximation is valid in the vicinity of the magical filling factors, i.e. at low $\mathrm{C}^{2} \mathrm{~F}$ density. The complete energy curve for the quantum-liquid phases would require a full account of these interactions, which is beyond the scope of the present studies.

The results for the cohesive energies of the different $\mathrm{CF}$ phases, given by the Eqs. (41), (5), and (7), are shown in Fig. 11 for three specific CF-LLs. Fig. 1(a) shows the results for CFs carrying 2 flux quanta $(s=1)$ in the first excited CF-LL $(p=1)$. The discussion is limited to the CF filling factor range $0<\bar{\nu}^{*}<1 / 2$, which corresponds to a range of the electronic filling factor $1 / 3<\nu<3 / 8$. In analogy with the electronic case, the range $1 / 2<\bar{\nu}^{*}<1$ is related to the shown part by the particle-hole symmetry. Our energy calculations suggest that a spin-polarized Laughlin-type quantum liquid of CFs has a lower energy than the competing $\mathrm{CF}$-solid phases around $\bar{\nu}^{*}=1 / 3$ and $1 / 5$, which correspond to $\nu=4 / 11$ and $6 / 17$, respectively. Especially the $4 / 11$ state is found to be largely favored, as expected from the form of the pseudopotentials;
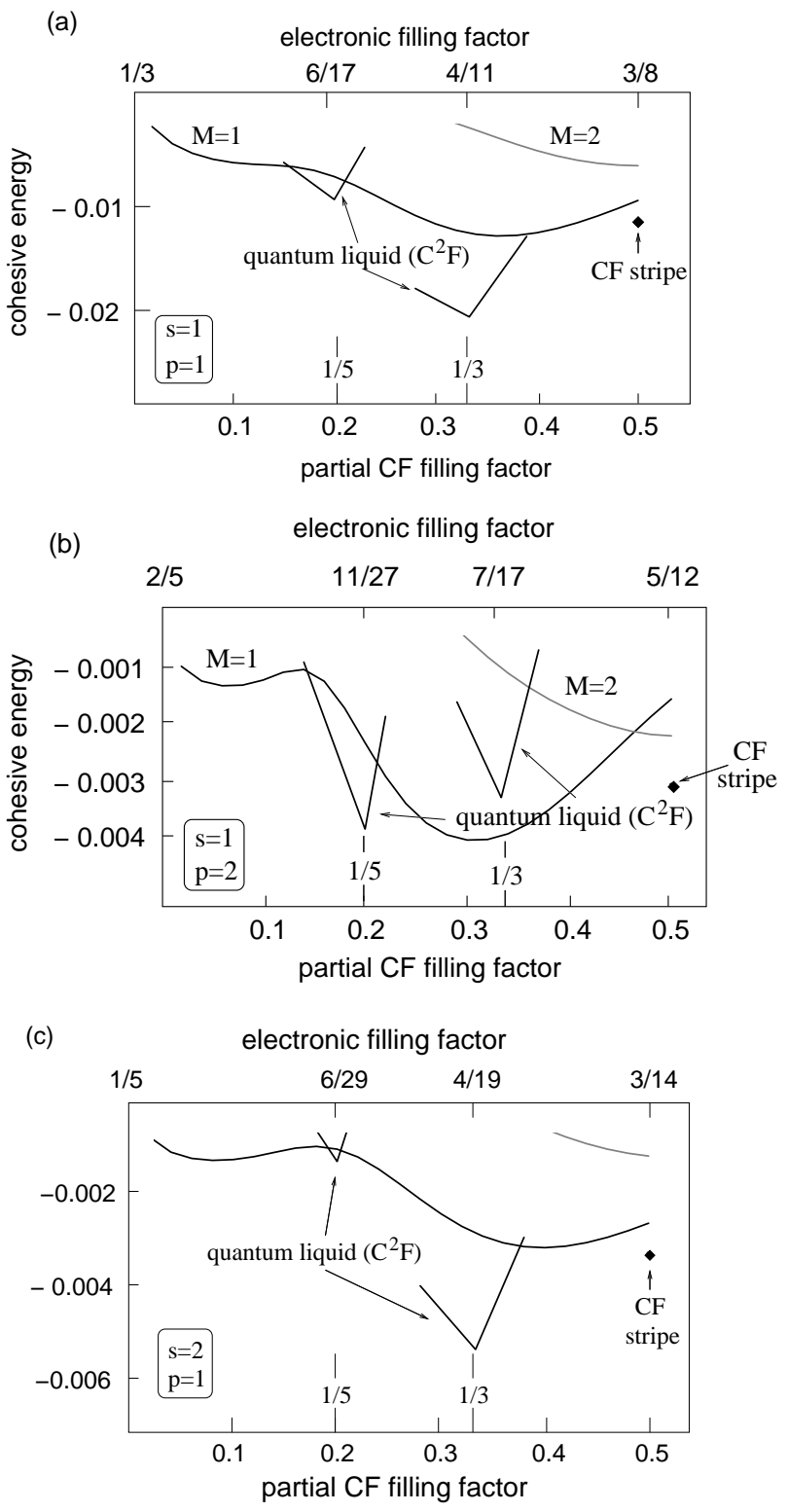

FIG. 1: Cohesive energies of the different CF phases for (a) $s=1, p=1$, (b) $s=1, p=2$, and (c) $s=2, p=1$ in units of $e^{2} / \epsilon l_{B}$.

one finds that $V_{3}(s=p=1)$ is smaller than its neighboring pseudopotentials [5]. This stabilizes the $\tilde{s}=1$ Laughlin state, which has its largest weight at the corresponding angular momentum and which screens $V_{1}$ completely [17]. Indeed, a spin-polarized FQHE at $\nu=4 / 11$ has been observed by Pan et al. 77]. The CF-WC becomes stable at lower densities, $\bar{\nu}^{*} \lesssim 0.15$, as well as at larger values $\bar{\nu}^{*} \gtrsim 0.4$. At $\bar{\nu}^{*}=1 / 2$, the CF-stripe phase has the lowest energy, which may lead to the anisotropic longitudinal resistance at $\nu=13 / 8$, recently observed by Fischer et al. 20. Contrary to the electronic case in the first excited LL 2], a CF-bubble crystal with two CFs 
$(M=2)$ per lattice site never has a lower energy than the CF-WC $(M=1)$. Although screening of the CF interaction potential affects the activation gaps [5] and may modify the transition points between the different phases, the investigated reentrance phenomenon persists in $1 / 3<\nu<2 / 5$. The effect of screening, finite sample width, and impurities on these phases will be discussed elsewhere 21].

For $s=1$ and $p=2$ [Fig.1(b)], i.e. in the range $2 / 5<$ $\nu<5 / 12$, the $\mathrm{CF}$ quantum liquid ceases to be the ground state at $\bar{\nu}^{*}=1 / 3$, where the CF-WC has the lowest energy. However, an incompressible $\mathrm{CF}$ liquid is found to be stable at $\bar{\nu}^{*}=1 / 5$. In contrast to the CF-LL $p=1$, a CF-bubble crystal with $M=2$ has a lower energy than the CF-WC around half-filling, but a CF-stripe phase is found to be the ground state at $\bar{\nu}^{*}=1 / 2$. For $1 / 5<\bar{\nu}^{*}<$ $3 / 14$, which corresponds to $p=1$ for CFs carrying 4 flux quanta $(s=2)$ [Fig. 1( (c)], a $\bar{\nu}^{*}=1 / 3$ Laughlin state of CFs is the lowest-energy state at $\nu=4 / 19$, whereas the liquid state is extremely close in energy to the CF-WC at $\bar{\nu}^{*}=1 / 5$. In the presence of impurities, which lower the energy of the solid phases more significantly than the quantum-liquid energies [2], a $\bar{\nu}^{*}=1 / 5$ Laughlintype state might vanish. Impurities may also affect the 6/17 state. In all cases an insulating CF-WC, which is the natural ground state at lower densities, has a lower energy also at rather large filling factors, $\bar{\nu} \gtrsim 0.4$. This may lead to reentrance phenomena, which would be the $\mathrm{CF}$ analogue of the reentrant IQHE observed at lower magnetic fields [1].

The stability of $\mathrm{C}^{2} \mathrm{~F}$ states has been a controversial issue during the last decade. Numerical investigations in the Haldane-Halperin hierarchy [17, 22] by Béran and Morf denied the stability of a spin-polarized 4/11 state, whereas they found a small gap in the absence of complete spin-polarization [23]. Their results have been confirmed by Wójs and Quinn 24] and by numericaldiagonalization studies in the $\mathrm{CF}$ wave-function approach by Mandal and Jain [10 and Chang et al. 25]. However, more recent studies by Chang and Jain 11], which hint to a stable 4/11 state, contradict their previous results. The size of the diagonalized system remains too small to allow for a conclusive answer of its stability in the thermodynamic limit.

In conclusion, we have calculated the energies of competing CF-solid and liquid phases in a recently developed model of interacting CFs [5]. Incompressible quantum liquids of spin-polarized CFs, which may be interpreted in terms of $\mathrm{C}^{2} \mathrm{Fs}[5,[6]$, are found to be stable at $\nu=4 / 11,6 / 17,4 / 19$, and $11 / 27$. Around half-filling of the topmost CF-LLs, a CF-stripe phase has the lowest energy, but may compete with a Pfaffian state [26], which has been omitted in the present studies. The fact that insulating CF-bubble crystals occur at different filling factors, which surround the CF-liquid phases, may lead to an experimentally observable reentrance of the FQHE in high-quality samples, in analogy to the reentrant IQHE in the first excited electronic LL [1].

We acknowledge fruitful discussions with R. Mani, R. Moessner, R. Morf, and A. Wójs. This work was supported by the Swiss National Foundation for Scientific Research under grant No. 620-62868.00.

[1] J. P. Eisenstein et al., Phys. Rev. Lett. 88, 076801 (2002).

[2] M. O. Goerbig, P. Lederer, and C. Morais Smith, Phys. Rev. B 68, 241302 (2003); ibid. 69115327 (2004).

[3] J. K. Jain, Phys. Rev. Lett. 63, 199 (1989); Phys. Rev. B 41, 7653 (1990).

[4] S.-Y. Lee et al., Phys. Rev. Lett. 87, 256803 (2001); Phys. Rev. B 66, 085336 (2002).

[5] M. O. Goerbig, P. Lederer, and C. Morais Smith, Phys. Rev. B 69, 155324 (2004); Europhys. Lett. 68, 72 (2004).

[6] A. Lopez and E. Fradkin, Phys. Rev. B 69, 155322 (2004).

[7] W. Pan et al., Phys. Rev. Lett. 90, 016801 (2003).

[8] R. G. Mani and K. v. Klitzing, Z. Phys. B 100, 635 (1996).

[9] G. Murthy and R. Shankar, Rev. Mod. Phys. 75, 1101 (2003); R. Shankar, Phys. Rev. B 63, 085322 (2001).

[10] S. S. Mandal and J. K. Jain, Phys. Rev. B 66, 155302 (2002).

[11] C.-C. Chang and J. K. Jain, Phys. Rev. Lett. 92, 196806 (2004).

[12] S. M. Girvin, A. H. MacDonald, and P. M. Platzman, Phys. Rev. B 33, 2481 (1986).

[13] A. A. Koulakov et al., Phys. Rev. Lett. 76, 499 (1996); M. M. Fogler et al., Phys. Rev. B 54, 1853 (1996).

[14] R. Moessner and J. T. Chalker, Phys. Rev. B 54, 5006 (1996).

[15] R. B. Laughlin, Phys. Rev. Lett. 50, 1395 (1983).

[16] A. H. MacDonald, Phys. Rev. B 30, 3550 (1984).

[17] F. D. Haldane, Phys. Rev. Lett. 51, 605 (1983).

[18] S. M. Girvin, Phys. Rev. B 30, 558 (1984).

[19] M. O. Goerbig and C. Morais Smith, Phys. Rev. B 66, 241101 (2002).

[20] F. Fischer et al., Physica E 22, 108 (2004).

[21] M. O. Goerbig, P. Lederer, and C. Morais Smith, unpublished.

[22] B. I. Halperin, Phys. Rev. Lett. 52, 1583 (1984).

[23] P. Béran and R. Morf, Phys. Rev. B 43, 12654. (1991).

[24] A. Wójs, J. J. Quinn, Phys. Rev. B 61, 2846 (2000).

[25] C.-C. Chang et al., Phys. Rev. B 67, 121305 (2003).

[26] G. Moore and N. Read, Nucl. Phys. B 360, 362 (1991); M. Greiter et al., Phys. Rev. Lett. 66, 3205 (1991); Nucl. Phys. B 374, 567 (1992). 